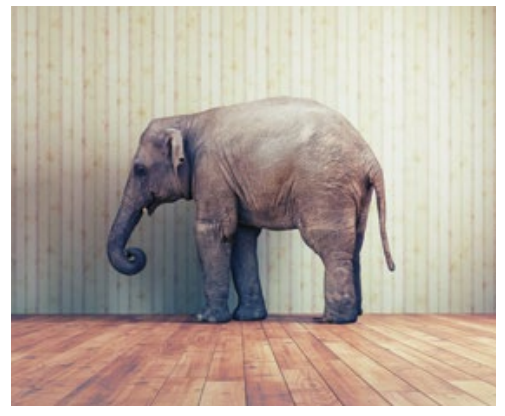

\title{
Primary care prevention of the cardiovascular health crisis for people with severe mental illnesses
}

\author{
The elephant in the room
}

Victoria J Palmer, Matthew Lewis,

Vasilios Stylianopolous, John Furler

\section{Background}

People living with psychosis, bipolar disorder, schizophrenia, major depressive disorders and other delusional disorders, collectively termed 'severe mental illness' (SMI), are at increased risk of dying prematurely from cardiovascular disease (CVD). The risk factors associated with this reduced life expectancy are largely modifiable.

\section{Objective}

The aim of this paper is to provide summative evidence of some of the known effective interventions that may reduce cardiovascular risk in people living with SMI.

\section{Discussion}

Pharmacological and nonpharmacological interventions are available and can be used in primary care for CVD risk reduction in people with SMI. Trials of pharmacological interventions for weight gain and smoking have shown good effects. Combined pharmacological and lifestyle interventions could support management of other risk factors, but tailored support and engagement strategies are needed. Specific guidance for CVD risk reduction in people with $\mathrm{SMI}$ is absent, but evidence indicates that following guidelines for the general population is warranted.
GIVEN OUR KNOWLEDGE of what can only be referred to as a cardiovascular health crisis for people living with severe mental illness (SMI), we propose that the old problem of the 'elephant in the room' has reared its head. This refers to a known problem that remains unspoken about in public, or 'an uncomfortable truth hidden in plain sight'. ${ }^{1}$ Psychosis, bipolar disorder, schizophrenia, major depression and other delusional disorders are complex conditions, commonly referred to in the literature by the umbrella term SMI. Although SMI affects a small proportion of the population (2-3\%), rates of cardiovascular disease (CVD) and metabolic syndrome in this group are 2.5 times higher than in the general community, ${ }^{2,3}$ and people with SMI under the age of 50 years are six times more likely to die from CVD. ${ }^{4}$ Since the 1980 s, there has been a steady decline in CVD in the general population. ${ }^{5}$ However, this has not been the case for people with SMI, ${ }^{6}$ who die 10-20 years earlier than the general population, ${ }^{2}$ a life expectancy gap almost equivalent to that faced by Aboriginal and Torres Strait Islander peoples. Preventive efforts are urgently needed, the problem is known yet persists; it is an uncomfortable truth.

Research has established that the reduced life expectancy for people living with SMI is not a direct result of mental illness or death by suicide, but it is largely due to preventable physical health conditions, particularly
CVD. ${ }^{2,7-9}$ Risk factors for CVD include abdominal obesity, insulin resistance/ glucose intolerance, hypertension, high cholesterol, physical inactivity, a high-fat diet, smoking, alcohol and other drug use, which are all over-represented in the SMI group. ${ }^{5}$ Antipsychotic medications are associated with a range of cardiovascular and metabolic abnormalities, such as obesity, hyperglycaemia, dyslipidaemia, and an increased risk of type 2 diabetes mellitus and CVD. ${ }^{10}$ The underlying mechanisms are unclear. Antipsychotic medications vary in the extent to which they influence cardiometabolic risk, although the association is most marked for olanzapine and clozapine..$^{10,11}$

Two recently completed randomised controlled trials (RCTs) emphasise an important role for primary care in tackling this major health problem. A US RCT found that a care management approach delivered by nurses in primary care improved preventive care and increased referrals to evidence-based services for cardiometabolic conditions in the intervention group. For those with available data, the Framingham Cardiovascular Risk Index reduced from baseline to 12-month follow-up in the intervention (7.8 to $6.9 \%$ ) while worsening in the control group (8.2 to 9.8\%). ${ }^{12}$ The PRIMROSE study, a UK cluster RCT of proactive nurse-delivered interventions in general practice to reduce cardiovascular risk, ${ }^{6}$ observed a reduction in total cholesterol over 12 months in 
both the intervention and control groups. There was no significant difference between groups, but hospitalisations decreased in the intervention group. ${ }^{6}$ There is a need for detailed evaluation of current interventions that could reduce CVD risk, further adaptation of this knowledge for people with SMI and support for implementation within primary care.

\section{The evidence for effective interventions to reduce CVD risk for people with SMI}

Since 2008, 16 systematic reviews have focused on the impact of pharmacological and non-pharmacological interventions on different aspects of cardiovascular health, CVD risk factors and/or other related physical health problems specifically for people living with SMI. Here, the evidence drawn from this literature is summarised by risk factor to provide a high-level overview of efforts in primary care that could address cardiovascular risk in people with SMI.

\section{Overweight and obesity}

The majority of efforts to reduce the risk of CVD in the SMI population relates to interventions that address weight and broadly support pharmacological and behavioural interventions to improve weight outcomes. ${ }^{13}$ A meta-analysis of pharmacological interventions for weight gain in people with SMI showed metformin to be effective (mean weight loss of $2.93 \mathrm{~kg}$; $95 \%$ confidence interval [CI]: 0.97, 4.89; $P=0.003) .{ }^{14}$ Three further reviews supported the use of metformin - and, to a lesser extent, topiramate - to reduce weight in people with SMI in trial settings. ${ }^{13,15,16}$ There was greater support for metformin than topiramate, as metformin appeared to be more widely studied and better tolerated and have a more favourable side-effect profile. ${ }^{16}$

Non-pharmacological interventions for weight loss, combining diet and exercise interventions, significantly reduced body mass index (BMI) and waist circumference. A meta-analysis of pooled results showed a weighted mean reduction of BMI at three-month follow-up of $-1.16 \mathrm{~kg} / \mathrm{m}^{2}$ (95\% CI: $-1.72,-0.58)$, increasing at six months to $-1.42 \mathrm{~kg} / \mathrm{m}^{2}$ (95\% CI: $\left.-1.83,-1.00\right)$, with reductions continuing at 12 months $\left(-2.03 \mathrm{~kg} / \mathrm{m}^{2} ; 95 \% \mathrm{CI}:-3.01,-1.05\right)$ and similar results in waist circumference to 12 months. ${ }^{17}$ A meta-analysis of data from trials of exercise therapy (including walking, general aerobic exercise and cycling) showed small but non-statistically significant reductions in BMI. ${ }^{18}$ Supervised exercise training within a multimodal program (which may include dietary modification, cognitive behavioural therapy or lifestyle education) reduced weight in people with SMI in one review ${ }^{19}$ but not in another. ${ }^{20}$ There was significant heterogeneity in the length, intensity and uptake of lifestyle interventions to reduce weight/BMI/waist circumference in reviewed studies, but programs that combined diet, lifestyle and physical activity components appeared most beneficial. ${ }^{17}$ Insufficient intensity and short duration of the intervention may have been factors in the inconsistency of these results.

\section{Smoking}

A systematic review of smoking cessation and reduction indicated that bupropion was more effective than placebo (relative risk [RR]: 3.03; 95\% CI: 1.69, 5.42) and had a beneficial effect in achieving abstinence at six months in people with schizophrenia (RR: 2.78; 95\% CI: 1.02, 7.58). ${ }^{21}$ In this review, varenicline was also more effective than placebo (RR: 4.74; 95\% CI: 1.34, 16.71); one trial followed participants to six months, but the confidence intervals were too great to infer sustained efficacy (RR: 5.06; 95\% CI: 0.67, 38.24). There was no evidence of benefit in quit rates or for smoking reduction in this population for other pharmacological therapies, including nicotine replacement therapy and psychosocial interventions. A comprehensive review of 20 studies determined that smoking reduction using bupropion and varenicline had the highest strength of evidence, with significant effects on both point prevalence and continued abstinence. ${ }^{13}$

\section{Glycaemia}

Antipsychotic switching, metformin and behavioural interventions have all been studied for their effect on glycated haemoglobin (HbA1c) and fasting blood glucose levels (BGL) in people with SMI. ${ }^{13,15,22,23}$ Findings were mixed across reviews, with a modest effect at best, although in many studies, glycaemia was a secondary endpoint rather than a study focus. ${ }^{13}$ Use of metformin had beneficial effects on $\mathrm{HbA} 1 \mathrm{c}$ in a comprehensive review of 33 studies $^{13}$ and in an additional systematic review of eight RCTs, ${ }^{15}$ but in both reviews it appeared to have no effect on BGL. Individual antipsychotics did not appear to improve $\mathrm{HbA1c}$, but treatment algorithms including switching to an atypical antipsychotic (eg aripiprazole, quetiapine or ziprasidone) used in combination with metformin found a statistically significant reduction in HbA1c. ${ }^{13,15,22}$

Taylor et al reviewed 54 studies and included 48 in a meta-analysis; they observed that both behavioural and pharmacological interventions reduced fasting BGL but had no impact on HbA1c. ${ }^{22}$ Longer behavioural interventions that included repeated physical activity had the greatest impact on fasting BGL, but there is limited evidence currently available to assess the impact of this type of intervention on HbA1c. Metformin and antipsychotic switching strategies were effective in lowering $\mathrm{HbA} 1 \mathrm{c}$ but did not have an effect on fasting BGL. ${ }^{22}$ Cabassa et al reviewed lifestyle interventions (eg behavioural interventions to improve diet or increase physical activity) for people with SMI. The review found that seven out of 13 studies reported statistically significant findings for lifestyle interventions to improve metabolic syndrome risk factors; however, some of these risk factors were secondary outcomes within studies. Two studies reported significant improvements in BGL or HbA1c. ${ }^{23}$

\section{Physical activity and cardiorespiratory fitness}

Interventions to improve cardiorespiratory fitness are largely beneficial, but some groups have reported mixed results. 
McGinty et al reported mixed effects on peak oxygen output and the six-minute walk test, ${ }^{13}$ but others have observed improvements in the six-minute walk test $^{18}$ and cardiorespiratory fitness. ${ }^{19,20}$ Improvements in cardiorespiratory fitness (as measured by $\mathrm{VO}_{2}$ max or $\mathrm{VO}_{2}$ peak, indicating the maximum rate of oxygen consumption during exercise and overall endurance capacity) have been shown with interventions including aerobic exercise, specifically those that are high intensity, high frequency (more than three times a week) and supervised by a qualified physiotherapist or exercise physiologist. ${ }^{19,20}$

\section{Blood pressure}

Most existing studies have included hypertension as a secondary endpoint to weight and may not be adequately powered to detect change in blood pressure. Only one review explicitly included hypertension as a category, and reported that the use of metformin and behavioural interventions to improve weight had no effect on blood pressure. ${ }^{13}$ Cabassa et al reported that a number of studies identified a significant impact on blood pressure following lifestyle intervention, but the results appear mixed. ${ }^{23}$

\section{Lipid profile}

Studies have typically included lipids as a secondary endpoint to weight and may lack statistical power to detect any changes to lipids. ${ }^{13,15}$ McGinty et al reviewed 27 papers that included a measure of hyperlipidaemia, and could only conclude that orlistat had no effect on lipids and that clinical screening interventions did improve lipid screening rates in SMI. ${ }^{13}$ There has been little conclusive evidence available from trials of pharmacological agents such as statins in this population aside from the recently completed PRIMROSE trial. ${ }^{6}$ Physical activity interventions, often with additional dietary advice or other educational programs, have shown moderate and sustained benefit in reducing triglyceride levels and total cholesterol over 12 months, but the evidence is limited. ${ }^{17}$

\section{Discussion}

\section{There is evidence to support action, so why does CVD risk in SMI remain unaddressed?}

While the evidence base examining CVD risk reduction in people with SMI is not extensive and is heterogeneous, it does indicate that pharmacological and non-pharmacological interventions may show promise for people living with SMI. The outcomes resoundingly support the need for a focused effort by the government and health system to address this crisis in a vulnerable, high-risk population. The results also indicate that effective interventions to reduce CVD risk in people with SMI shared common factors with best practice CVD risk prevention for the general population. It may, however, take longer for people with SMI to engage in interventions and change behaviour, ${ }^{13}$ making it essential that long-term programs be implemented and evaluated and that engagement strategies are inbuilt. Primary care is the ideal setting to address cardiovascular health in people with SMI, as general practitioners (GPs) can actively screen for CVD risk in their patients, conduct ongoing follow-up, modify treatment, refer on as indicated and engage nurses to assist in tackling this challenge. The CVD crisis in people with SMI will be the focus of a funded National Health and Medical Research Council (NHMRC) RCT to deliver a proactive and assertive care-informed intervention in primary care in 2018-22.

There has been a prominent view that people with SMI do not access primary care; however, recent Australian figures from the national Survey of High Impact Psychosis showed that $88 \%$ of people with SMI visited a GP, with an average frequency of nine times per year, compared with five visits per year in the general population. ${ }^{24}$ This frequency of contact with GPs presents an invaluable opportunity to engage patients with SMI in efforts to work longitudinally to improve their cardiovascular health. Delivering multifactorial interventions in primary care to the general community can be complex, and this complexity is amplified in the SMI population, as outlined in Figure 1.
Figure 1 illustrates the need for multilevel interventions in the healthcare setting and indicates that there are major challenges to reach this population, including internalised stigma, feeling unwelcome in healthcare environments and having mental illness overshadow physical health needs. Additionally, a critical gap exists in current guidance; despite strong evidence linking CVD risk to SMI, people with SMI are not highlighted prominently as a high-risk population. ${ }^{2}$ The Royal Australian College of General Practitioners' Smoking, nutrition, alcohol, physical activity guide refers to the inequitable burden of CVD risk factors in higher risk populations such as Aboriginal and Torres Strait Islander people, people who are refugees and those of low socioeconomic background, but does not specifically refer to people with SMI. Some individual risk factors, such as smoking, identify people with SMI as a high-risk group, and the role of atypical antipsychotics is discussed in relation to weight gain. Alcohol is also identified as an exacerbating factor in mental illness. ${ }^{25}$ Similarly, the Guidelines for preventive activities in general practice and Putting prevention into practice: Guidelines for the implementation of prevention in the general practice setting, designed to support preventive care in general practice, do not link an increased risk of CVD with SMI, but recognise that mental illness can act as a barrier to accessing care generally. ${ }^{26,27}$

\section{Limitations as they apply to Australian general practice}

While evidence exists that CVD risk in people with SMI can be responsive to some treatments, these results must be interpreted with some important caveats: many RCTs have been conducted at single sites or in hospital and outpatient settings; the majority have addressed single risk factors and few measure absolute CVD risk; non-pharmacological interventions were highly variable in terms of mode and length of delivery; there is considerable heterogeneity within the populations studied; and few studies have been conducted in the Australian primary care setting. These factors limit the generalisability of the reviewed evidence 
and necessitate that more research be conducted to determine effective strategies to reduce CVD risk in people with SMI.

\section{Conclusion}

The reviews included in this paper all present interventions that align with guidelines for clinical best practice for their respective treatment targets, but modest effect sizes relative to the general population suggest that tailored, SMI-specific interventions should be developed..$^{22}$ It should be noted that moderate effects are still meaningful as they could delay the onset of diabetes, diabetes-related complications or CVD. ${ }^{22}$ However, in the absence of SMI-specific treatment recommendations, clinicians should aim to achieve care to the level of general population guidelines to address the increased burden of CVD in people with SMI. ${ }^{15}$ Broadly speaking, there is evidence to suggest that longer-term, intensive interventions that combine both pharmacological and non-pharmacological components alongside behavioural and lifestyle interventions targeting diet and physical activity will be the most effective in treating CVD risk in people with SMI. This approach will best address the specific challenges such as those outlined in Figure 1. This figure is a helpful reminder that in primary care we must better identify the needs of people living with SMI and tailor interventions to the person's social and personal circumstances. The role of GPs is not only to consider what effective interventions can be delivered, but also to engage relationally so that these interventions may be configured within

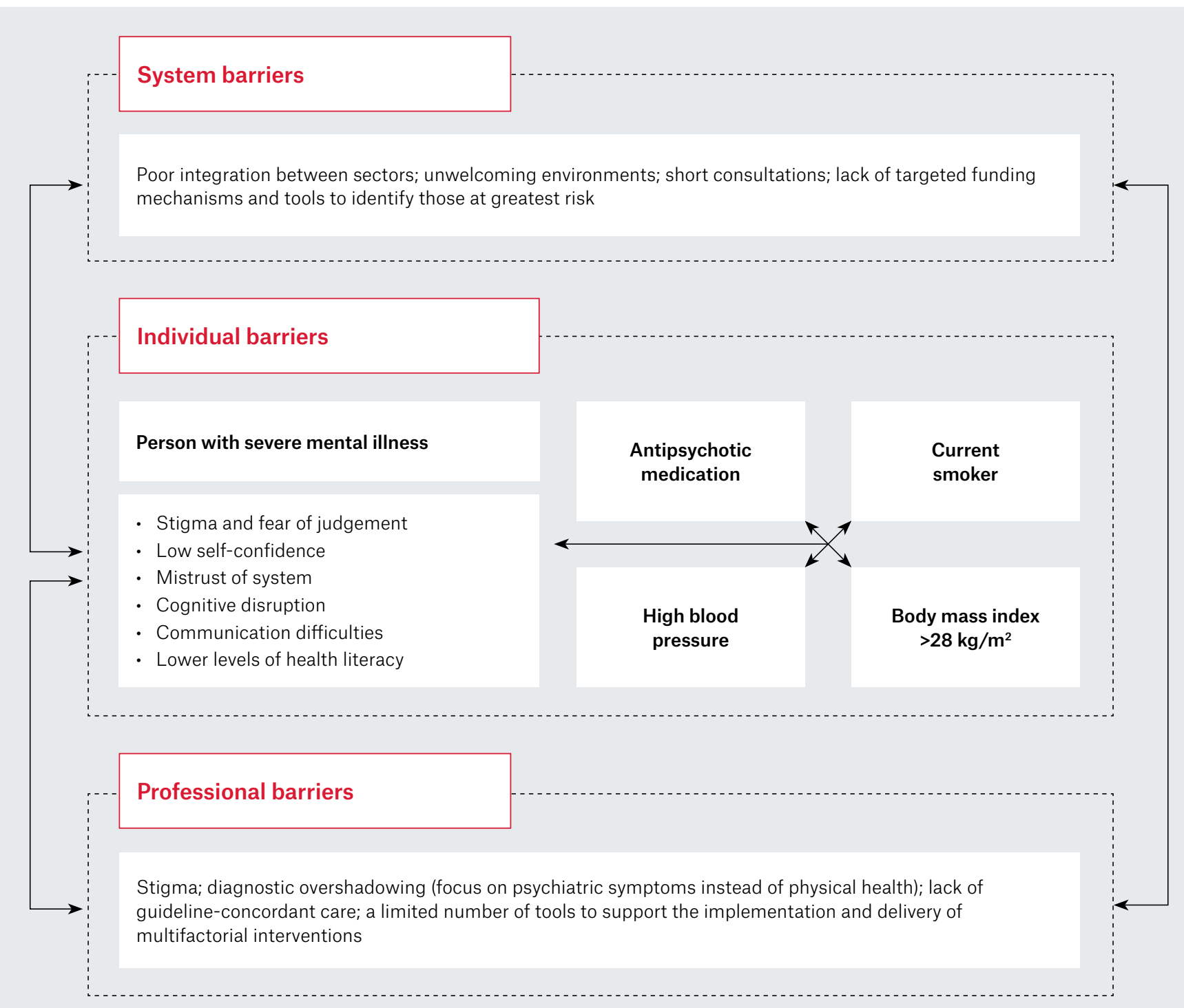

Figure 1. A synthesis of known challenges to addressing physical health needs for people living with severe mental illness 
the life circumstances and needs of the person at high risk. Many people will have multiple risk factors to address, thus finding ways to support multifactorial approaches within the complexity of living with mental illnesses is critical in primary care.

\section{Authors}

Victoria J Palmer PhD, BA(Hons), Senior Research Fellow, Honorary Fellow Network Society Institute (Applied Ethics), Department of General Practice, Melbourne Medical School, Faculty of Medicine, Dentistry and Health Sciences, University of Melbourne, Vic.vpalmer@unimelb.edu.au Matthew Lewis PhD, Research Fellow, Department of General Practice, Melbourne Medical School, Faculty of Medicine, Dentistry and Health Sciences, University of Melbourne, Vic

Vasilios Stylianopolous, Medical student, Department of General Practice, Melbourne Medical School, Faculty of Medicine, Dentistry and Health Sciences, University of Melbourne, Vic

John Furler BMMS, GradDip Public Health, PhD, FRACGP, Associate Professor, Department of General Practice, Melbourne Medical School, Faculty of Medicine, Dentistry and Health Sciences, University of Melbourne, Vic

Competing interests: None.

Funding: The work is funded by an NHMRC Project Grant 2018-2022 (APP1141344) 'Assertive care: A randomised controlled trial to reduce cardiovascular risk for people with severe mental illness'. The named investigator team for the grant includes: Dr Victoria Palmer, Professor Jane Gunn, Professor Carol Harvey, Associate Professor John Furler, Professor David Osborn, Dr Sandra Davidson, Dr Mahesh Jayaram Dr Patty Chondros, Professor Amanda Kenny. Associate investigators: Professor Mark Nelson, Professor Stephen Harrap, Professor David Castle Professor Vera Morgan, Professor Stewart Mercer. Provenance and peer review: Commissioned, externally peer reviewed.

\section{Acknowledgements}

The authors acknowledge research support provided by Ms Kali Godbee and the integrated mental health research program.

\section{References}

1. Zerubavel E. The elephant in the room: Silence and denial in everyday life. Oxford: Oxford University, 2006.

2. Liu NH, Daumit GL, Dua T, et al. Excess mortality in persons with severe mental disorders: $A$ multilevel intervention framework and priorities for clinical practice, policy and research agendas. World Psychiatry 2017;16(1):30-40. doi: 10.1002/ wps.20384

3. Morgan VA, McGrath JJ, Jablensky A, et al. Psychosis prevalence and physical, metabolic and cognitive co-morbidity: Data from the second Australian national survey of psychosis. Psychol Med 2014;44(10):2163-76. doi: 10.1017/ S0033291713002973.

4. Osborn DJ, Levy G, Nazareth I, Petersen I, Islam A, King MB. Relative risk of cardiovascular and cancer mortality in people with severe mental illness from the United Kingdom's general practice research database. Arch Gen Psychiatry 2007;64(2):242-49. doi: 10.1001/ archpsyc.64.2.242.
5. Galletly CA, Foley DL, Waterreus A, et al. Cardiometabolic risk factors in people with psychotic disorders: The second Australian national survey of psychosis. Aust N Z J Psychiatry 2012;46(8):753-61. doi: 10.1177/0004867412453089.

6. Osborn D, Burton A, Hunter R, et al. Clinical and cost-effectiveness of an intervention for reducing cholesterol and cardiovascular risk for people with severe mental illness in English primary care: A cluster randomised controlled trial. Lancet Psychiatry 2018;5(2):145-54. doi: 10.1016/S22150366(18)30007-5.

7. The Royal Australian and New Zealand College of Psychiatrists. The economic cost of serious mental illness and comorbidities in Australia and New Zealand. Melbourne: RANZCP, 2016.

8. World Health Organization. Addressing comorbidity between mental disorders and major noncommunicable diseases. Geneva: WHO, 2017.

9. De Hert M, Cohen D, Bobes J, et al. Physical illness in patients with severe mental disorders. II. Barriers to care, monitoring and treatment guidelines, plus recommendations at the system and individual level. World Psychiatry 2011;10(2):138-51.

10. Chang SC, Lu ML. Metabolic and cardiovascular adverse effects associated with treatment with antipsychotic drugs. J Experimental Clin Med 2012;4(2):103-07.

11. De Hert M, Detraux J, van Winkel R, Yu W, Correll CU. Metabolic and cardiovascular adverse effects associated with antipsychotic drugs. Nat Rev Endocrinol 2011;8(2):114-26. doi: 10.1038/ nrendo.2011.156.

12. Druss BG, von Esenwein SA, Compton MT, Rask KJ, Zhao L, Parker RM. A randomized trial of medical care management for community mental health settings: The Primary Care Access, Referral, and Evaluation (PCARE) study. Am J Psychiatry 2010;167(2):151-59. doi: 10.1176/appi. ajp.2009.09050691.

13. McGinty EE, Baller J, Azrin ST, Juliano-Bult D, Daumit GL. Interventions to address medical conditions and health-risk behaviors among persons with serious mental illness: A comprehensive review. Schizophr Bull 2016:42(1):96-124. doi: 10.1093/schbul/sbv101.

14. Fiedorowicz JG, Miller DD, Bishop JR, Calarge CA, Ellingrod VL, Haynes WG. Systematic review and meta-analysis of pharmacological interventions for weight gain from antipsychotics and mood stabilizers. Curr Psychiatry Rev 2012;8(1):25-36. doi: 10.2174/157340012798994867.

15. Gierisch JM, Nieuwsma JA, Bradford DW, et al. Pharmacologic and behavioral interventions to improve cardiovascular risk factors in adults with serious mental illness: A systematic review and meta-analysis. J Clin Psychiatry 2014;75(5):e42440. doi: 10.4088/JCP.13r08558.

16. Ellinger LK, Ipema HJ, Stachnik JM. Efficacy of metformin and topiramate in prevention and treatment of second-generation antipsychoticinduced weight gain. Ann Pharmacother 2010;44(4):668-79. doi: 10.1345/aph.1M550.

17. Fernández-San-Martín MI, Martín-López LM, Masa-Font R, et al. The effectiveness of lifestyle interventions to reduce cardiovascular risk in patients with severe mental disorders: Metaanalysis of intervention studies. Community Ment Health J 2014;50(1):81-95. doi: 10.1007/s10597013-9614-6.

18. Pearsall R, Smith DJ, Pelosi A, Geddes J. Exercise therapy in adults with serious mental illness: A systematic review and meta-analysis. BMC Psychiatry 2014;14:117. doi: 10.1186/1471-244X-14-117.
19. Chalfoun C, Karelis AD, Stip E, Abdel-Baki A. Running for your life: A review of physical activity and cardiovascular disease risk reduction in individuals with schizophrenia. J Sports Sci 2016;34(16):1500-15. doi: 10.1080/02640414.2015.1119875.

20. Vancampfort D, Rosenbaum S, Schuch F, et al. Cardiorespiratory fitness in severe mental illness: A systematic review and meta-analysis. Sports Med 2017;47(2):343-52. doi: 10.1007/s40279-0160574-1.

21. Tsoi DT, Porwal M, Webster AC. Interventions for smoking cessation and reduction in individuals with schizophrenia. Cochrane Database Syst Rev 2013;(2):CD007253. doi: 10.1002/14651858. CD007253.pub3.

22. Taylor J, Stubbs B, Hewitt C, et al. The effectiveness of pharmacological and nonpharmacological interventions for improving glycaemic control in adults with severe mental illness: A systematic review and meta-analysis. PLoS One 2017;12(1):e0168549. doi: 10.1371/ journal.pone.0168549.

23. Cabassa LJ, Ezell JM, Lewis-Fernández R. Lifestyle interventions for adults with serious mental illness: A systematic literature review. Psychiatr Serv 2010;61(8):774-82. doi: 10.1176/ps.2010.61.8.774.

24. Morgan VA, Waterreus $A$, Jablensky $A$, et al. People living with psychotic illness in 2010: The second Australian national survey of psychosis. Aust N Z J Psychiatry 2012;46(8):735-52. doi: 10.1177/0004867412449877.

25. The Royal Australian College of General Practitioners. Smoking, nutrition, alcohol, physical activity (SNAP): A population health guide to behavioural risk factors in general practice. East Melbourne, Vic: RACGP, 2015.

26. The Royal Australian College of General Practitioners. Guidelines for preventive activities in general practice. 9th edn. East Melbourne, Vic: RACGP, 2016.

27. The Royal Australian College of General Practitioners. Putting prevention into practice: Guidelines for the implementation of prevention in the general practice setting. 3rd edn. East Melbourne, Vic: RACGP, 2018. 